\title{
Discrepâncias de assentamento ocorridas em copings cerâmicos confeccionados pelos sistemas IPS Empress 2 e In-Ceram, variando-se os términos cervicais e os agentes cimentantes: estudo in vitro
}

\section{Adjustment discrepancies in ceramic copings made in IPS Empress and In-Ceram systems, varying the cervical end and the cements used: an in vitro study}

\author{
Virgílio Vilas Boas FERNANDES JR \\ Mestre - Departamento de Odontologia Restauradora - Faculdade de Odontologia de São José dos Campos - UNESP - \\ São José dos Campos - SP - Brasil.
}

\section{Aldari Raimundo FIGUEIREDO \\ Carlos Augusto PAVANELLI \\ Prof. Doutor - Departamento de Materiais Odontológicos e Prótese - Faculdade de Odontologia de São José dos Campos - UNESP - São José dos Campos - SP - Brasil.}

\section{Lafayette NOGUEIRA JR}

Prof. Adjunto - Departamento de Materiais Odontológicos e Prótese - Faculdade de Odontologia de São José dos Campos - UNESP - São José dos Campos - SP - Brasil.

\author{
Aldari FIGUEIREDO \\ Estagiário em Periodontia - Departamento de Diagnóstico e Cirurgia - Faculdade de Odontologia de São José dos Campos \\ - UNESP - São José dos Campos - SP - Brasil.
}

\begin{abstract}
Resumo
Este trabalho avaliou as adaptações cervicais de copings cerâmicos, confeccionados pelos Sistemas Empress 2 e In-Ceram, variando-se os términos cervicais dos preparos em chanfro largo e ombro $90^{\circ}$ arredondado; em preparos com $15^{\circ}$ de conicidade, e 2 tipos de agentes para cimentação: o cimento de fosfato de zinco e um cimento resinoso. Foram confeccionados dois modelos-padrão de aço inoxidável. A partir destes obtiveram-se 40 troquéis em gesso tipoV, sendo 20 para cada sistema e 10 para cada tipo de término cervical e os copings cerâmicos correspondentes para cada sistema. Em seguida, o conjunto foi levado ao metroscópio horizontal para a primeira leitura (L0). Procedeu-se a cimentação, inicialmente sob pressão digital, em seguida com a aplicação de carga padronizada e efetuou-se a segunda medida (L1). Após a coleta dos dados, foi realizada a análise estatística das médias das medidas aferidas e as seguintes conclusões foram alcançadas: a) o cimento de fosfato apresentou um valor médio de discrepância de assentamento, para os dois sistemas, menor do que o resinoso; b) término cervical em chanfro e ombro arredondado, para qualquer um dos sistemas, mostraram resultados semelhantes; c) o sistema In-Ceram apresentou um valor médio de discrepância assentamento menor que o IPS Empress 2, porém não significante estatisticamente.
\end{abstract}

\section{UNITERMOS}

Términos cervicais; adaptação marginal; cimentação; cerâmica.

\section{INTRODUÇÃO}

A adaptação marginal é um critério importante usado para avaliação clínica das peças protéticas cimentadas. A presença de desajuste marginal em coroas totais pode promover o contato do agente de cimentação com o meio bucal. Quanto maior for esta desadaptação, mais rapidamente poderá ocorrer a dissolução do cimento, permitindo um incremento na retenção de placa bacteriana e, em conseqüência, aumento da irritação aos tecidos periodontais e pulpares, comprometendo a longevidade da restauração protética e do dente suporte ${ }^{10}$. 
A dimensão da desadaptação marginal das restaurações fixas é dependente dos vários passos envolvidos no processo clínico e laboratorial, podendo ser traduzida como uma somatória de distorções, que são inerentes a cada etapa de confecção e das características dos materiais empregados ${ }^{5}$.

Segundo alguns autores ${ }^{6,15}$ a estética em coroas metalocerâmicas ou de cerâmica pura, poderá apresentar-se prejudicada, devido a presença do metal nas primeiras ou de cimentos radiopacos nas últimas, fatores esses limitantes à transmissão da luz.

A necessidade estética da era contemporânea norteou a introdução dos novos sistemas de coroas totalmente cerâmicas, dentre eles destacam-se os sistemas IPS Empress 2 e In-Ceram.

Mesmo com todo o desenvolvimento dos sistemas restauradores livres de metal, a incompatibilidade de união dos sistemas cerâmicos com as estruturas dentinárias tem sido alvo de grande preocupação e estudo. Cimentos convencionais como o fosfato de zinco e de ionômero de vidro têm sido utilizados na cimentação dessas cerâmicas, contudo a alta solubilidade e a força de adesão desses cimentos às estruturas dentais são menores que a dos sistemas adesivos resinosos. A dificuldade e sensibilidade da técnica de cimentação, porém, ainda têm limitado a utilização desses sistemas.

Com relação ao término cervical dos preparos realizados para receberem coroas totais em cerâmica pura, os mais adequados, segundo os próprios idealizadores dos sistemas, seriam o chanfro largo e o degrau em $90^{\circ}$ arredondado, condições estas que ofereceriam melhor estética, resistência e ajuste marginal ${ }^{2}$.
Diante do exposto, o objetivo desse trabalho é verificar as discrepâncias de assentamento ocorridas em copings cerâmicos confeccionados pelos sistemas IPS Empress 2 (Ivoclar Vivadent Ltda, São Paulo, SP, Brasil) e In-Ceram (Vita, Wilcos do Brasil, Petrópolis, RJ, Brasil), variando-se os términos cervicais e os agentes cimentantes.

\section{Material e Método}

Cilindros de aço inoxidável (V303) com diâmetro de $12,70 \mathrm{~mm}$ foram usinados em torno $\mathrm{CNC}$, produzindo corpos-de-prova com as seguintes características:

- altura dos preparos $-5,5 \mathrm{~mm}$;

- diâmetro maior dos preparos - 8mm;

- diâmetro maior das paredes axiais $-6,4 \mathrm{~mm}$;

- conicidade total dos preparos $-15^{\circ}\left(7,5^{\circ} \mathrm{em}\right.$ cada parede axial);

- término cervical - chanfro largo e ombro $90^{\circ}$ arredondado

Posteriormente, a posição do cilindro foi invertida no torno e abriu-se internamente na sua base não desgastada uma cavidade com rosca de $7 \mathrm{~mm}$, com a finalidade de adaptá-lo e posicioná-lo à haste móvel de um delineador modificado, especialmente idealizado, para realização das moldagens individuais, sempre nas mesmas condições operacionais. $\mathrm{Na}$ margem cervical do preparo coronário realizou-se um entalhe para permitir o exato reposicionamento dos copings cerâmicos nos troquéis de trabalho, bem como no modelo padrão (Figura 1).

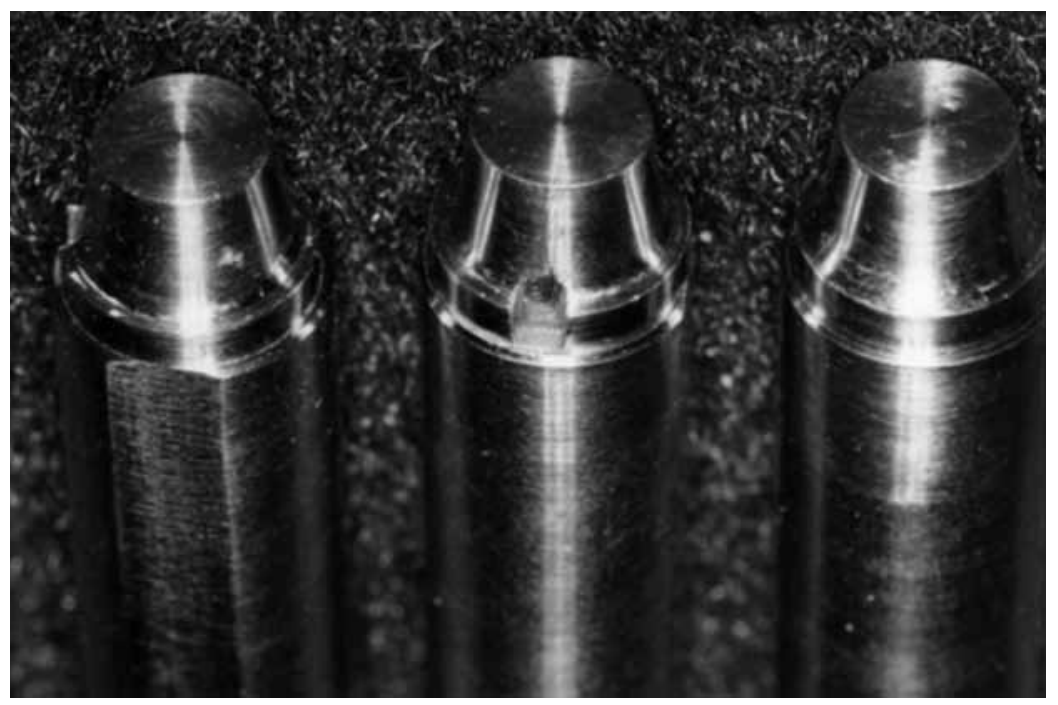

Figura 1 - Vista dos modelos-padrão representativos de preparos para coroa total preconizados para próteses em cerâmica vítrea, em aço inoxidável. Observar encaixe em uma das faces. 
Realizou-se a reprodução do modelo padrão por meio de moldagens, empregando-se uma silicona de adição (Elite H-D Putty Soft - Normal Setting e Regular Body - Normal Setting, Zhermack, Itália) pela técnica da dupla moldagem, em moldeiras individuais confeccionadas em aço Villares SAE 5115, que era encaixada na base do delineador. Por meio da haste móvel do delineador modificado, o modelo-padrão pôde ser fixado e posicionado de modo constante. A profundidade de inserção do modelo padrão no material de moldagem foi determinada por uma mola localizada na porção superior da haste (Figura 2). Para a obtenção de alívio padronizado no material denso, um casquete de polietileno com espessura de $0,8 \mathrm{~mm}$ foi previamente prensado a vácuo e adaptado nos corpos-de-prova, obtendo-se um padrão para a pré-moldagem com o material denso.

Aguardaram-se-se no mínimo duas horas após a moldagem para obtenção de 40 troquéis de trabalho em gesso pedra especial tipo IV (Durone, Dentsply, Petrópolis, RJ, Brasil).

Foram confeccionados vinte copings cerâmicos do Sistema IPS Empress 2 e vinte no sistema In-Ceram.

O coping foi assentado no modelo-padrão (Figura 3) e em seguida, fez-se atuar a carga de $5 \mathrm{Kg}$ sobre ele, durante quatro minutos. Decorrido esse período, o conjunto, ainda sob a carga de $5 \mathrm{~kg}$, foi travado por meio de um dispositivo específico ${ }^{9}$. Posteriormente, o conjunto foi levado ao metroscópio horizontal para a primeira leitura, que convencionamos chamar de LO.

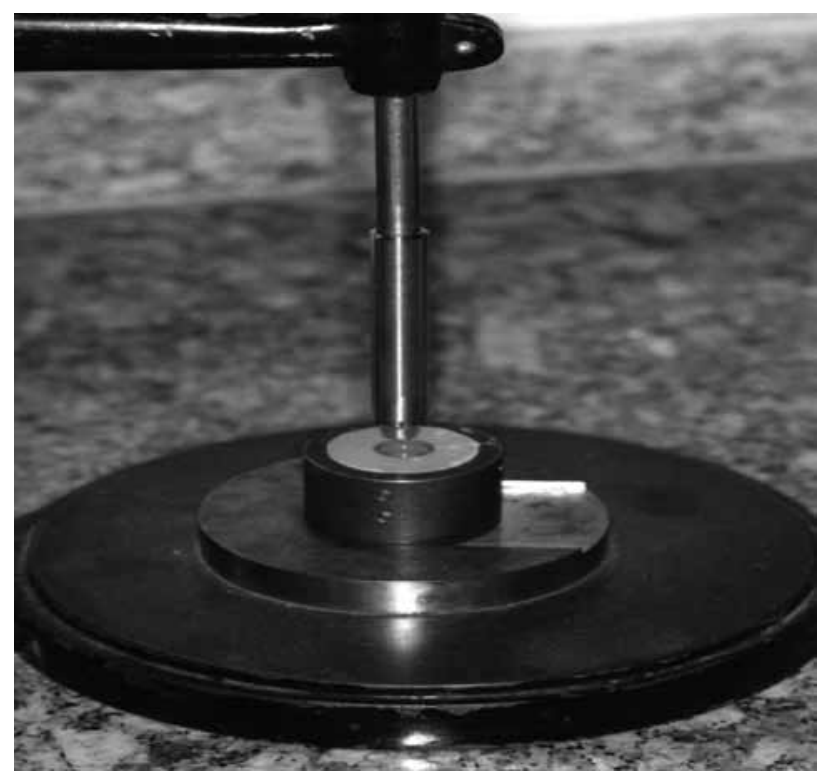

Figura 2 - Vista do delineador modificado para obtenção da moldagem do modelo-padrão sempre na mesma posição e moldeira (porção inferior).
Após a leitura L0, foi preparado o cimento resinoso Variolink II Esthetic Cementation System (Ivoclar Vivadent, São Paulo, SP, Brasil), seguindo-se rigidamente as recomendações do fabricante. Ele foi inserido no interior do coping sendo posicionado e assentado no modelo-padrão, primeiro sob pressão digital e depois sob uma carga de $5 \mathrm{Kg}$ por 4 minutos. O corpo-deprova foi mensurado, como no item anterior para a medida L0. Estes procedimentos foram executados dez vezes, para cada sistema, obtendo-se assim as medidas denominadas L1 para cada coping cimentado.

Para a manipulação do cimento fosfato de zinco (S.S.White Artigos Dentários, Rio de Janeiro, RJ, Brasil), antes de sua espatulação, o pó foi dividido em quatro partes iguais. Uma das partes foi dividida em duas partes de um oitavo, e em seguida uma destas partes foi dividida ao meio, em duas partes de 1/16, segundo recomendações de Figueiredo ${ }^{4}$, Bottino ${ }^{1} \mathrm{e}$ Rocha et al. ${ }^{11,12}$.

A mistura foi pincelada no interior do coping, que em seguida foi posicionado no modelo-padrão sob pressão digital e depois sob carga de $5 \mathrm{Kg}$ por 4 minutos. O conjunto foi levado ao metroscópio horizontal para mensuração e obtenção da medida L1. Estes procedimentos foram executados dez vezes para cada sistema, obtendo-se assim medidas denominadas L1, para cada coping cimentado.

Fazendo-se a subtração L1 menos L0 obteve-se a discrepância de assentamento, ocorrida para cada tipo de corpo-de-prova e para cada cimentação.

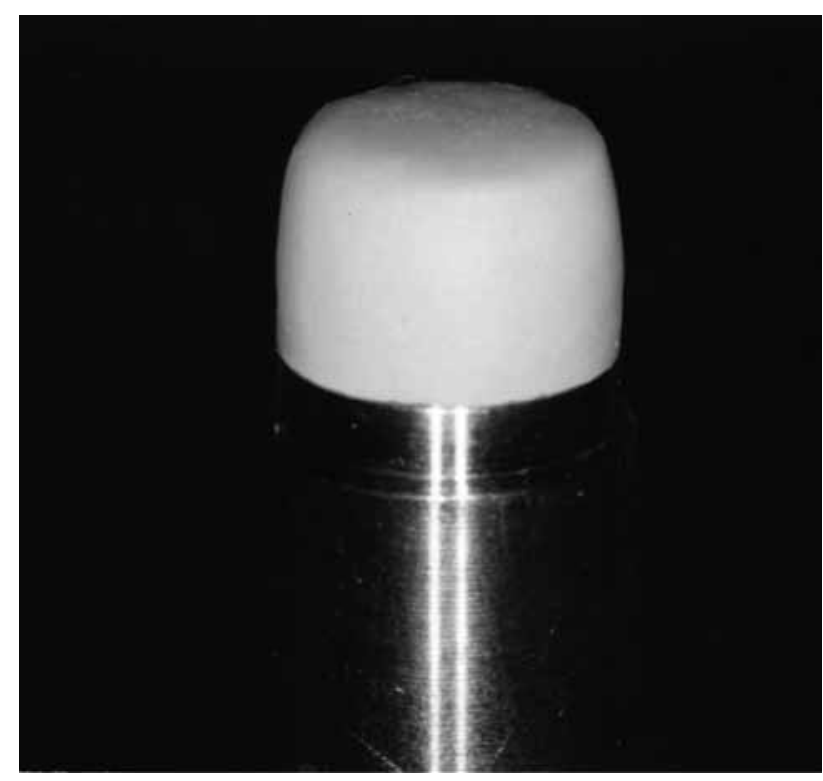

Figura 3 - Vista do coping assentado sobre o modelo-padrão. 


\section{Resultados e Discussão}

Os dados obtidos foram submetidos à análise de Variância (ANOVA) para testar a existência de diferenças significativas nos desajustes médios (discrepâncias) para as variáveis propostas. Quando após a aplicação da ANOVA verificou-se um efeito das variáveis significante, efetuou-se o teste de comparação múltipla de Tukey. O nível de significância adotado foi sempre o valor convencional de $5 \%$.
Inicialmente considerou-se o conjunto das variáveis testadas, ou seja: os cimentos selecionados; os términos dos preparos; e os sistemas empregados na confecção dos copings e as situações de mensuração L0 e L1.

Pode-se observar, na Tabela 1 , o resultado da ANOVA 3 fatores.

Tabela 1 - Dados da ANOVA para discrepância de assentamento $\left(L_{1}-L_{0}\right)$

\begin{tabular}{lccccc}
\hline Efeito & GI & SQ & QM & F & p-valor \\
\hline Cimento & 1 & 0,00193 & 0,00193 & 17,28 & $0,0002^{*}$ \\
Sistema & 1 & 0,000318 & 0,000318 & 2,86 & 0,1005 \\
Término & 1 & 0,000000702 & 0,00000070 & 0,01 & 0,9372 \\
Cimento x Sistema & 1 & 0,000000735 & 0,00000018 & 6,60 & $0,0151^{*}$ \\
Cimento x Término & 1 & 0,000000 & 0,000187 & 0,00 & 0,9680 \\
Sistema x Término & 1 & 0,000187 & 0,0000217 & 1,68 & 0,2044 \\
Cimento x Sistema x Término & 1 & 0,0000217 & 0,000111 & 0,20 & 0,6616 \\
Resíduo & 32 & 0,00357 & & & \\
Total & 39 & 0,00675 & & & \\
\hline
\end{tabular}

* diferença estatisticamente significante, $\mathrm{p}<0,05, \mathrm{GI}$ : grau de liberdade; SQ: soma dos quadrados; QM: quadrado médio

Verifica-se, mediante o resultado da ANOVA, que podemos considerar como significante o efeito do fator principal cimento, bem como, o efeito interação entre essa variável (cimento) e o sistema (material restaurador). Com respeito ao fator interação cimento x sistema, utilizou-se o teste de comparação múltipla de Tukey (5\%), para identifi- carmos grupos homogêneos de mesmo desempenho, conforme apresentado na Tabela 2. Observa-se nas médias desta interação (cimento $\mathrm{x}$ sistema) que a condição experimental cimento resinoso / sistema Empress 2 apresenta um valor médio de desajuste estatisticamente diferente das demais condições experimentais.

Tabela 2 - Teste de Tukey (5\%) para a variável interação cimento x sistema. Dados em $\mathrm{mm}$.

\begin{tabular}{lccc}
\hline Cimento & Sistema & Médias & Grupos homogêneos* \\
\hline Resinoso & Empress & 0,0763 & $\mathrm{~A}$ \\
Resinoso & In-Ceram & 0,0621 & $\mathrm{~B}$ \\
Fosfato & In-Ceram & 0,0568 & $\mathrm{~B}$ \\
Fosfato & Empress & 0,0538 & $\mathrm{~B}$ \\
\hline
\end{tabular}

* Médias seguidas de letra iguais, na mesma coluna, não diferem estatisticamente.

Quando consideramos os sistemas, os términos de maneira independente, e ainda, a interação cimento $\mathrm{x}$ término, sistema $\mathrm{x}$ término e cimento $\mathrm{x}$ sistema $\mathrm{x}$ término, os resultados não são significativos estatisticamente $(p>0,05)$.

Observa-se que quando se empregou o cimento resinoso para o sistema Empress 2 pode-se considerar o resultado como significante.
Dentre os fatores que influenciam a longevidade protética, pode-se destacar a adaptação marginal ou justeza do limite cervical, representada teoricamente pela determinação de uma situação de continuidade anatômica e estrutural, verificada nas áreas limítrofes entre o dente preparado e o componente protético, determinando-se que haja apenas uma linha de separação entre esses elementos, sem que se evidencie qualquer tipo de separação entre eles. 
O tipo de desenho do término cervical é o que determina esta perfeita integridade, é ele que fornecerá espessura e forma adequadas do material restaurador na margem da restauração.

Muito se tem discutido na literatura sobre qual seria o tipo de término ideal que pudesse favorecer o assentamento das restaurações, proporcionando uma linha de desajuste cervical mínima, aceitável do ponto de vista clinico. Alguns autores estudaram a influência da configuração marginal no desajuste de coroas $^{4,10,11,12,14}$. Neste trabalho, devido às características estruturais dos materiais cerâmicos, utilizamos os términos preconizados pelos fabricantes dos Sistemas Empress 2 e In-Ceram e indicados por Bottino et al. ${ }^{2}$.

Quintas et al. ${ }^{10}$ concluíram em seus estudos que o término cervical do preparo influenciou no desajuste cervical antes da cimentação, porém não foi significativo após a mesma. Em nossos estudos, analisando-se o efeito término do preparo isoladamente, independentemente das outras variáveis, ficou evidenciando que não houve diferença estatística significativa entre os dois tipos de término.

Quando se analisou em nossos estudos a interação término x sistema, observou-se que o término em chanfro mostrou-se ideal para ambos os sistemas (In-Ceram 78,210 $\pm 0,1284 \mu \mathrm{m}$ e IPS Empress 79,051 $\pm 0,2519 \mu \mathrm{m}$ ), assim como o ombro arredondado (InCeram 78,251 $\pm 0,1220 \mu \mathrm{m}$ e IPS Empress 79,145 \pm $0,2747 \mu \mathrm{m})$, ao contrário do que concluíram Oliveira et al. ${ }^{8}$, que verificaram que o sistema In-Ceram com ombro arredondado apresentou o pior resultado médio em comparação com o sistema In-Ceram em chanfro, Procera e Empress 2 em chanfro e ombro arredondado.

Para McLean e Von Fraunhofer ${ }^{7}$, pequena expulsividade do preparo dificulta o escoamento do agente cimentante na linha ocluso-axial, refletindo diretamente no valor do desajuste marginal. Em nossos estudos optamos por preparos com conicidade de $15^{\circ}$, o que é mais recomendado para as coroas estéticas livres de metal ${ }^{1}$.

É importante determinar o tipo do agente cimentante que irá preencher a interface da superfície do dente preparado e a face interna da restauração. Deve-se considerar as características deste cimento, para avaliar-se o possível grau de desajuste cervical, após a cimentação final da prótese. Avaliou-se neste trabalho a quantidade de discrepância marginal antes e após a cimentação e quando se analisou o efeito cimento separadamente nas situações L0 e L1, in- dependentemente dos efeitos sistema e término dos preparos, pode-se observar diferença estatisticamente significativa, para o cimento de fosfato de zinco em L0 $(78,613 \pm 0,4492 \mu \mathrm{m})$ em L1 $(78,669 \pm 0,4489 \mu \mathrm{m}) \mathrm{e}$ para o cimento resinoso em L0 $(78,652 \pm 0,5244 \mu \mathrm{m})$ e em L1 $(78,722 \pm 0,5319 \mu \mathrm{m})$, estando de acordo com Campbell ${ }^{3}$ (1990), que afirmou a introdução do agente de fixação altera a adaptação obtida sem a presença do mesmo. Apesar da avaliação inicial ser importante, é o desajuste final que afeta a longevidade das restaurações protéticas ${ }^{4}$.

Quando se analisou o efeito cimento na discrepância de assentamento dos quarenta copings cerâmicos no modelo padrão, independentemente da variável material restaurador e término do preparo, observou-se que o cimento de fosfato apresentou um valor médio $(0,0553 \pm 0,0104 \mathrm{~mm})$ estatisticamente inferior ao cimento resinoso $(0,0692 \pm 0,0121 \mathrm{~mm})$.

Quando se observou a interação cimento x período ( $\mathrm{L} 0$ e L1) x sistema, independentemente do tipo de término, pode-se deduzir que para o sistema In-Ceram qualquer um dos cimentos empregados, independentemente do término, mostra-se bastante eficaz.

Empregamos uma carga estática de $5 \mathrm{Kgf}$ durante 4 minutos, tanto na situação sem agente de cimentação, como após a cimentação, ao contrário do que preconizam Rosenstiel e Gegauff ${ }^{13}$ que estudaram a influência da aplicação de cargas dinâmicas, afirmando que isso resulta em menor desajuste cervical, comparativamente à aplicação de cargas estáticas. Outro fator muito importante a ser considerado seria a viscosidade do agente de fixação, pois cimentos mais viscosos exigem uma maior força de assentamento das coroas, o que poderia induzir estresse nas áreas cervicais das coroas em cerâmica pura durante $o$ ato de cimentação.

As discrepâncias cervicais das próteses cerâmicas livres de metal têm relacionamento extremamente importante com o resultado clínico dos tratamentos protéticos, portanto todos os estudos e medidas que visem uma redução deste desajuste refletirão positivamente na manutenção e longevidade destas restaurações.

Não existe um cimento ideal para toda situação clínica. É absolutamente necessário que se avalie caso a caso para que se possa usufruir melhor das propriedades de cada agente cimentante, observando de maneira crítica as suas principais características, vantagens e indicações, antes de utilizá-lo. 


\title{
Conclusões
}

Analisando-se os resultados deste estudo pode-se concluir que:

a) O cimento de fosfato apresentou um valor médio de discrepância de assentamento, para os dois sistemas, menor do que o cimento resinoso; b) Término cervical em chanfro e o ombro arredondado mostraram resultados semelhantes;

c) O sistema In-Ceram apresentou um valor médio de discrepância assentamento menor que o IPS Empress 2, porem não significante estatisticamente.

\begin{abstract}
The aim of this study was to assess the cervical adjustments of ceramic copings, using two ceramic systems, the Empress 2 and In-Ceram, varying the cervical end of the preparations and the types of cements: zinc phosphate and resin cement. Initially two standard models of stainless steel were made. From these models 40 stone dies were made, 20 for each system and 10 for each type of cervical end. A specialized commercial laboratory made the ceramic copings corresponding to each system. Then, the set was brought to the horizontal metroscopic for first measure, called L0. The cementing process was done, initially under digital pressure, and then with the implementation of standardized load, when the second measure, called L1, was done. After collecting the data, statistical analysis was carried out. We could conclude that- a) zinc phosphate cement showed less settlement discrepancy, for both systems, than the resin cement b) rounded shoulder and chamfer ends showed similar results, c) In-Ceram system had less settlement discrepancy, but not statistically significant, than IPS Empress 2.
\end{abstract}

\section{UNITERMS}

Cervical end; marginal fit; cements; ceramics.

\section{REFERÊNCIAS}

1. Bottino MA. Avaliação in vitro da adaptação cervical de coroas totais metálicas, variando os preparos dos términos cervicais, aliviando ou não as superfícies internas das coroas e empregando diferentes cimentos definitivos [livre docência]. São José dos Campos: Faculdade de Odontologia de São José dos Campos, Universidade Estadual Paulista "Júlio Mesquita Filho"- UNESP; 1998

2. Bottino MA. Cimentação em próteses livres de metal. In: Estética em reabilitação oral: metal free. São Paulo: Artes Médicas; 2001. cap7. p.381-441.

3. Campbell S. Comparison of conventional paint-on die spacers and those used with the all-ceramic restorations. J Prosthet Dent. 1990;63:151-5.

4. Figueiredo AR. Discrepâncias de assentamento ocorridas em coroas totais metálicas, com e sem alívio interno, variando-se os términos cervicais e os agentes cimentantes [tese]. São José dos Campos: Faculdade de Odontologia de São José dos Campos, Universidade Estadual Paulista "Júlio Mesquita Filho" - UNESP; 1997.

5. Gassino G, Barone MS, Scanu M, Spina G, Preti G. Marginal adaptation of fixed prosthodontics: anew in vitro 360-degree external examination procedure. Int J Prosthodont. 2004 mar-apr;17(2):218-23.

6. Kelly JR, Nishimura AI, Campbell SD. Ceramics in dentistry: historical roots and current perspectives. J Prosthetic Dent. 1996;75(1):18-32.

7. Mclean JW, Vonfraunhofer JA. The estimation of cement, film thickness by in vivo technique. Br Dent J. 1971;131:107-11.

8. Oliveira AA, Saito T. Oliveira SHG. Adaptação marginal de copings de três sistemas cerâmicos em função de dois tipos diferentes de terminação cervical. Rev Ciênc Ext. 2007;3(2):39.

9. Pavanelli CA. Discrepância vertical de assentamento de coroas totais: dispositivo para fixação e mensuração pré- e pós cimentação (in vitro). Pós Grad Rev Fac Odontol São José dos Campos. 2001;4(2):60-4.
10. Quintas AF, Oliveira F, Bottino MA. Vertical marginal discrepancy of ceramic copings with different ceramic materials finish lines, and luting agents: an in vitro evaluation. J Prosthetic Dent. 2004;92(3):250-7.

11. Rocha CAJ, Figueiredo AR, Bottino MA. Discrepância vertical de coroas cimentadas: nova metodologia para sua verificação e análise. Rev Odontol UNESP. 1998;27(1):39-51.

12. Rocha CAJ, Figueiredo AR, Bottino MA. Análise das discrepâncias verticais verificadas em coroas totais cimentadas, em função do tipo de acabamento cervical dos preparos, alívio interno e cimentos empregados. Pós-Grad Rev Fac Odontol São José dos Campos. 1998;1(1):35-46.

13. Rosenstiel SF, Gegauff AG. Improving the cementation of complete cast crowns: comparison of static and dynamic seating methods. JADA. 1988;117(7):845-8.

14. Shearer B, Gouch MB, Setchell DJ. Influence of marginal configuration and porcelain addition on the fit of In-Ceram crowns. Biomaterials. 1996;17(19):1891-5.

15. Touati B, Quintas AF. Aesthetic and adhesive cementation for contemporary porcelain crowns. Pract Proced Aesth Dent. 2001;13(8):611-20.

Recebido em 19/09/2008

Aprovado em 19/03/2010

Correspondência:

Virgílio Vilas Boas Fernandes Jr Endereço: Rua Enseada - 46 Bairro: Cidade Jardim CEP: 12230-630 São José dos Campos - SP e-mail: virgiliovilas@hotmail.com 